\title{
3 Research Suare \\ K-Clustering for the Recognition of Moving Entity by Wireless Sensor Networks Using Deep Learning
}

Thiyagarajan R ( $\square$ thiagarajan82r@gmail.com )

Prathyusha Engineering College

\section{Balajivijayan V}

Vignan's Foundation for Science Technology and Research

\section{Rajalakshmi D}

RMD Engineering College

\section{Research Article}

Keywords: K-Clustering Technique, Deep Learning Algorithm, Wireless Sensor Networks, Moving Entity, Pervasive Computing

Posted Date: September 27th, 2021

DOl: https://doi.org/10.21203/rs.3.rs-913118/v1

License: (c) (i) This work is licensed under a Creative Commons Attribution 4.0 International License. Read Full License 


\section{Abstract}

For the detection of the moving entity, a deep learning algorithm is used. To detect the complex type of situations, real-time monitoring of a moving object has to be detected. Using the deep learning method, wireless sensor networks are diagnosed using virtual representation features. Using the k-clustering technique, it achieves the target detection and can determine the positioning. It can determine the characteristics based on the precision. The wireless sensor networks are proposed and analyzed by means of a statistical approach. Statistical way of clustering up of the data detects the precision rate and its positioning state. The positioning method eventually reduces the error which accumulates the efficiency. The main advantages are the robustness and efficiency to improve the performance. These moving targets can be detected with less computational cost. In the field of pervasive computing, the recognition of a moving target can be improvised. The sensor node transmits the information, i.e., communication to nodes. In this paper, the researches focus on wireless sensor data along with a moving entity. This research is mainly used in healthcare and Al-based applications. By using the loT with wireless sensor networks, the detection of a moving entity can be determined by using the combination of k-clustering algorithms. This deep learning algorithm reduces the time complexity and determines integrity in data.

\section{Introduction}

The loT develops the infrastructure of the moving entity target using recognition. These are newly developed pervasive computing techniques which develop the automatic way of identifying the research. Over the moving entity many different researchers have developed regarding the following entity. These can be used in the healthcare industry to monitor the patients. For the medical supplies and for the patient monitoring purpose, these Rfid tags can be used. To identify if there is any abnormality in crowded places or any hurdles for the moving entity. These can be used in many different applications for the athlete to identify their speed limit, blood pressure and other behavior analysis of the athletes. This is an important role in people's life moving entity by detecting it. Even in today's applications, a remote sensor identifies the object set by a smart home sensor. We can avoid the hurdles and give the necessary protection over the safety of the moving entity. This can be determined by detecting the image representation off data from different sources. The camera is used to monitor the moving object. Data processing is carried to analyze the image data. For the sensor, a wearable type sensor can be used to identify the data such as image, layout. Based upon the data on the image which are determined from the gyroscope, Rfid reader, wireless sensor, Bluetooth the data can be analyzed.

The data which are acquired using the image can be able to detect the primary over the data by theft in data confidentiality. Personal privacy over the information can be initiated to avoid the data theft. Al based human activity recognition can be able to recognize regularization over time, accuracy and reduces the complexity. Some of the achievements such as human-based recognition in terms of gait analysis, behavioral based analysis, and health monitoring management are determined. To monitor the physical and other environmental conditions, the wireless sensor networks are self- configured. This passes the 
data over the network of devices. This deploys a large number of sensors with the way of processing in an ad-hoc manner. It identifies the network of devices that communicate their information using wireless links. It implements security which imposes limitations of resources such as battery, bandwidth, efficiency, pervasive computing and memory. The range of deterministic attacks can focus on data privacy, data availability and control. WSN can be used in healthcare applications to monitor the patients. Rather than using a manual way of extraction, deep learning is used to classify the data set. The high level sensor is used along with a deep learning approach to identify the precise and accurate level of hurdles while moving entities. For the recognition of a moving entity, the deep learning algorithm can be used. This paper focuses on the research based upon WSN to detect the moving entity. The wearable sensor detects the data by collecting it, and the data are processed for a statistical data report. The statistical report features the different parameters of the sampled data. By using the K clustering technique, the entire sample data are being clustered for the further processing stage. Rather than using the traditional way of features, the different techniques of deep learning techniques are initiated to improve the efficiency and accuracy.

The hierarchical algorithm determines the way of standardized set of data samples in a hierarchical manner. Rather than using the hierarchical technique, the k-clustering technique can be used to target and send the image of the data set. The recognition of the fine-grained indicates high precision, consumption of low energy, robustness in state. Constant monitoring of the data detects the hurdles or any suspicious activity which avoids the unwanted disruptions. The large number of data is generated has to be implemented using the Al technology to avoid less complexity in data. By using the loT with wireless sensor networks, the detection of a moving entity can be determined by using the combination of $k$ clustering algorithms. This deep learning algorithm reduces the time complexity and determines integrity in data. Each type of motion is characterized according to the behavioral analysis. These tasks characterize the data by tracking the multiple objects in the surrounding environment. These types of applications can be used to derive the traffic mechanism, congestion over the network traffic, security surveillance system and other human-interactive security measures. It features the data by the deterministic way of number of clusters. The deep learning algorithm is used to extract the relevant source of information which consists of unknown regularity and an unknown set of patterns. Deep learning techniques are used nowadays to improve the efficiency over the fully automated and statistical outcome events.

\section{Background Work}

Linghua Zhao [1] proposed paper which determines the problems and issues which are faced in real time application detection where it traces out the location of the moving objects which are in complex situations. The researcher proposed a paper which initiates the novel approach of determining the target motion detection. The deep learning method is used where the visual representation of data is extracted from the offline similarity ranking function. The author also used model incremental in online state by using the hierarchical clustering technique. By using the hierarchical clustering technique, the detection of the target and its positioning can be determined. The histogram way of categorizing the data is by 
achieving the exact positioning of the object. The large set of comparative analysis is analyzed by a certain set of advantages and disadvantages. Both the qualitative and quantitative are used to derive the statistical approach. Rather than using traditional way of approach, deep learning approach is implemented. These generally use the less computational cost and have significant of high robustness.

Dong Hye Ye [5] proposed a novel technique about the Unmanned Aerial Vehicles which is used in military and civil applications. The development of collision can be avoided such as critical state in a crowded state. By using the UAV payload, the cost and limitations are stabilized. By using the video surveillance, the UAV can be able to identify the moving detection. Tracking algorithm has been used to detect the proper board efficiently. To track and detect UAV which is mounted by a single camera on it. The author proposed a way that estimates the transformation over the identification of a moving object. Deep learning determines the classification over the trained datasets such as labeled datasets. For each moving object, the spatio-temporal traits are characterized to determine the optical flow in matching. Even in kalman filter is used on moving objects, prune which can improve the temporal consistency. From the UAV, the algorithm used to validate the video datasets. By using the small track over the UAV, along with the limited computing resources, results are determined.

Supreeth H S G [6] proposed a way that the track over the object such as computer vision over the surveillance. Correlations are detected using the object tracking and detection components. The steps are performed to avoid the complication tasks. Deep neural network is used as a way to determine the learning over the multiple ways of representation. The abstraction over the data such as text, image are detected. Gaussian Mixture Model uses the detection of objects where the deep learning paradigm is used for the recognition over the tracking of objects. By using the following videos, the efficiency is improved. Here, the performances are analyzed using True Positive and False Alarm metrics. The author got the performance of about $88 \%$.

Azhee Wria Muhamad [2] proposed a way that the multiple movements of object detection such as surveillance systems. In this, traditional k-algorithm is used to develop speed and optimize the feature extraction. For the better accuracy, the F1 score is measured to develop the better accuracy and precision. Both the precision and recall accuracy is used for the computation of the score. The results are indicated such as it improves the accuracy and quality.

\section{Methodology}

\subsection{Wireless Sensor Networks}

A wireless sensor network inter links two or more devices to communicate with each other. These devices are connected using the internet medium. They usually use the internet service provider to determine the connectivity among each other. By using IEEE 802.11 WLAN, which has the standardized market standard, is used to connect the devices. These sensor networks use both the statistical and behavioral approach. By determining the actual set of devices which put up the data where it amplifies the signal 
using the wifi medium. They are based upon the infrastructure which is to monitor the physical, other environmental conditions to identify the detection. The monitored data from the sensor detects whether any abnormality is present while the sensor detection takes place. The base station is considered as an inter link to communicate between two nodes such as the user and network. These wsn uses gps to locate its positioning using the following algorithm to determine the exact location. It uses the standardized process to communicate with each other. Here, the application protocol domain is restricted by setting a certain set of privileges to it. Based upon the coverage and sensitivity, the data optimizations are done busing the connectivity.

\subsection{Behavioral Analysis}

The object detection has to be analyzed by using the situation interactive model. The collection of interaction of entities defines the well-defined in terms of a set of behaviors. The set of behavior gives the attributes with the interaction. The agreement is based on the structure of the result. The methods determine the classes based on the behavior. The modification of data determines the functionality of data. The subroutine defines the object class, without methods. The detection of a moving object is to recognize the movement of a physical and other movement of the entity. The stationary entity or movement entity is achieved by the structure by tracking it. Generally, the moving entity is determined using the cross-correlating evaluation based on the movable object. It determines the centre point along with the axis for the tracking of the entity. The tracking of data indicates the speed, accuracy, attribute and tracking of the positioning location. These can be used for patient's monitoring in the healthcare industry to identify the hurdles for the disability patient. These are automatically detected by the WSN sensor which alerts by using behavioral analysis of data. With the wearable sensor, the daily activity of the patient's data can be gathered.

\subsection{Moving Entity}

The wearable wireless network sensor detects the data using the video surveillance. It stores the data; the data are retrieved for later purpose. The moving object has to be detected for the positioning of the object. By using the location positioning method, the trace out of the position takes place. These moving entities are used in the health care industry and other organizations. These are similar to the smart home sensors, but the accuracy over the performance is comparatively high as it uses a deep learning algorithm to attain the precise result.

\subsection{K-Clustering Algorithm}

To partitioning the data into classes and clusters, the clustering has to be used. The clusters differentiate the object based on the similarity of the pattern and position. It detects the low and high rank index to identify the similarity. A framework is used to detect the clustering up of the moving entity of the object. These trajectories are determined by using the k-means clustering algorithm. This minimizes the average squared type of distance where it points out the distance between the points in the same cluster. It seeks to identify the similar positioning of the object. Inbuilt heuristic method is used to choose the number of clusters. With the initialization of the segment, the positioning of spatial are attained. In using this, the 
refinement can increase the accuracy. Euclidean distance is used to compute the Centroid cluster and the segment. It tests along with the previous segment for generating the new clusters with an initial set of Centroid. The Centroid is used to recalculate the segment by using the new positioning of the segment. The spatial positions are calculated using the average segments.

$$
J=\sum_{j=1}^{k} \sum_{i=1}^{n}\left\|\mathrm{x}_{\mathrm{i}}{ }^{\mathrm{j}=1}-\mathrm{c}_{\mathrm{j}}\right\|^{2}
$$

Where $\mathrm{j}$ indicates the function of objectivity, $\mathrm{K}$ denotes the number of clusters, $\mathrm{C}$ denotes the Centroid of the functionality.

$\left\|x_{i}{ }^{j=1}-c_{j}\right\|^{2}$ denotes the distance function between two different clusters to compute with it.

Step 1: Initialize the number of clusters K.

Step 2: Choose the random set of points such as from $\mathrm{K}$.

Step 3: Now, assign the data points according to the closest set of Centroid to the new form of clusters.

Step 4: After assigning it, compute the new Centroid for each cluster.

Step 5: Now, reassign the data point into the new set of Centroid clusters.

\section{Construction}

\subsection{Raw Data}

The data which are not processed are the raw data. The raw data which are processed are attained using the information and data. Huge numbers of the data are cleansed for the further purpose. Parsing method is used to identify the data by reforming and translating the data. The raw data holds up all the unwanted data without any filters. After the cleansing and parsing the data, it can be used for a further use. The data which is processed consists of outliners and other fine-grained format. If the data are not cleansed, the data might consist of risk which can cause exploitation over the data. By filtering the data, the cooked data can be accessed for the further processing stage.

\subsection{Data Collection}

The data which are designed are said to be raw data which are collected from the sensors. Wireless sensor networks assign the raw data which are collected. The data are collected using the data collection perspective. The informative processing and solving of the problem are raw data tasks. The tasks which are assigned are from the raw data. The data are recorded and stamped for the behavioral analysis.

\subsection{Data Preprocessing}


The data which are collected are processed for further purpose. The data are pre-processed at certain regular intervals. Based upon the video and image processing, the results are checked by determining the regular speech rate, time intervals, frequency and etc. The hidden pair of activity image recognition can be analyzed using the time series signals.

\subsection{Data Validation}

The processed data are validated for the further purpose. The validated data are formatted for the deterministic data. The data are simulated from the image classification and analyzed for the behavioral analysis. Based upon the behavioral analysis, the data are analyzed to monitor the suspicious activity of the entity. If the moving entities are suspicious or hurdle activity has to be detected. The validated data are represented by using the recorded feature and video processing.

\subsection{Analyzing Data set}

After validating the data, the data are further processed for analyzing. The data are analyzed to identify the behavioral analysis. The data are processed to identify the source, destination, primary, secondary, type of device to detect the moving entity. The positioning axis of the data is determined by deriving the exact position of the entity.

\subsection{Statistical Report}

The statistical report indicates the way in which they are driven is in an efficient way. Statistical way represents the way the data are handled. Determining the image by recognizing accordingly as per the approach. The approach is carried out in a proper sequence of analysis. The detection of an entity by analyzing the hurdles to avoid accidents by comparing it. The compared data are analyzed to determine the result outcome by using the k-clustering algorithm.

\section{Experimental Results \\ 5.1 Clustering of the dataset}

The dataset are clustered using the following steps. By segmentation of the data, the Centroid and the number of clusters can be determined. The use of silhouette is to reach the positive value, negative value of the clustering of the data. The data are divided in proper initialization without the empty clusters. The clusters identify and split up the data according to the similarity in the data

\subsection{Extracting the known values}




\begin{tabular}{|lllllll|}
\hline Dataset & $\begin{array}{l}\text { Number of } \\
\text { clusters }\end{array}$ & $\begin{array}{l}\text { Segmentation } \\
\text { Rate }\end{array}$ & $\begin{array}{l}\text { Time } \\
\text { Interval } \\
\text { (sec) }\end{array}$ & $\begin{array}{l}\text { Location } \\
\text { Time(sec) }\end{array}$ & $\begin{array}{l}\text { Object } \\
\text { Size(pixels) }\end{array}$ & $\begin{array}{l}\text { Image } \\
\text { Range }\end{array}$ \\
\hline $\begin{array}{l}\text { Object } \\
1\end{array}$ & 1000 & $89 \%$ & 0.65 & 0.45 & 18 & 3245 \\
\hline $\begin{array}{l}\text { Object } \\
2\end{array}$ & 1400 & $88 \%$ & 0.77 & 0.43 & 15 & 4567 \\
\hline $\begin{array}{l}\text { Object } \\
3\end{array}$ & 1300 & $97.3 \%$ & 0.86 & 0.67 & 12 & 7889 \\
\hline $\begin{array}{l}\text { Object } \\
4\end{array}$ & 1250 & $94 \%$ & 0.96 & 0.78 & 34 & 8757 \\
\hline
\end{tabular}

From the extraction of known values from the object, the different parameters are evaluated. By using the scatter graph, the parameters of the moving object are parameterized. Each object differs in their clusters and the segmentation rate. From the above-mentioned results, the object 3 attributes are high when compared to the other intervals. Based upon the segmentation of the data, the clustering of the data along with the Centroid is attained.

\section{Conclusion}

Thus, in this paper it focuses on the moving entity using the wireless sensor networks by classifying the data set in a statistical way. The statistical reports are determined using the k-clustering technique. The kclustering technique derives the cluster of data of the sampling dataset. By using the $\mathrm{K}$ clustering technique, the entire sample data are being clustered for the further processing stage. By using the loT with wireless sensor networks, the detection of a moving entity can be determined by using the combination of k-clustering algorithms. The deep learning algorithm is used to extract the relevant source of information which consists of unknown regularity and an unknown set of patterns. Statistical way of clustering up of the data detects the precision rate and its positioning state. Personal privacy over the information can be initiated to avoid the data theft. Al based human activity recognition can be able to recognize regularization over time, accuracy and reduces the complexity.

\section{Declarations}

\section{Funding}

Not applicable

\section{Conflicts of interest/ Competing interests}

The authors declare that this manuscript has no conflicts of interest with any other published source and has not been published. No data have been fabricated to support our conclusion. 


\section{Consent for Publication}

I confirm that this work is original and has not been published elsewhere nor it is currently under consideration for publication elsewhere. Thank you for your consideration of this manuscript.

\section{Authorship contributions}

All authors each made a significant contribution to the research reported and have read and approved the submitted manuscript.

\section{References}

1. Zhao, Linghua \& Huang, Zhihua. (2021). A Moving Object Detection Method Using Deep LearningBased Wireless Sensor Networks. Complexity. 2021. 1-12. 10.1155/2021/5518196.

2. Wria, Azhee \& Mustafa, Luqman \& Sabir, Ari. (2020). Multi Moving Object detection system using Modified K-means and Deep Learning Algorithms algorithm. Cihan University-Erbil Scientific Journal. 4. 18-32. 10.25098/4.1.23.

3. Zhu, Juncai \& Wang, Zhizhong \& Wang, Songwei \& Chen, Shuli. (2020). Moving Object Detection Based on Background Compensation and Deep Learning. Symmetry. 12. 1965. $10.3390 /$ sym 12121965 .

4. R \& Moorthi. M “Energy consumption and network connectivity based on Novel-LEACH-POS protocol networks",Computer Communications, Elsevier, (0140-3664), vol.149, pp. 90-98, November 2019.

5. Zhao, P. Zheng, S. Xu and X. Wu, "Object Detection With Deep Learning: A Review," in IEEE Transactions on Neural Networks and Learning Systems, vol. 30, no. 11, pp. 3212-3232, Nov. 2019, doi: 10.1109/TNNLS.2018.2876865.

6. Ye, Dong Hye \& Li, Jing \& Chen, Qiulin \& Wachs, Juan \& Bouman, Charles. (2018). Deep Learning for Moving Object Detection and Tracking from a Single Camera in Unmanned Aerial Vehicles (UAVs). Electronic Imaging. 2018. 4661-4666. 10.2352/ISSN.2470-1173.2018.10.IMAWM-466.

7. S. G. Supreeth and C. M. Patil, "Moving object detection and tracking using deep learning neural network and correlation filter," 2018 Second International Conference on Inventive Communication and Computational Technologies (ICICCT), 2018, pp. 1775-1780, doi: 10.1109/ICICCT.2018.8473354.

8. R \& Moorthi.M "Quality of Service Based Adhoc Ondemand Multipath Distance Vector Routing Protocol in Mobile AD HOC Network", Journal of Ambient Intelligence \& Humanized Computing , Springer, vol.12, issue no. 5, pp. 4957-4965 April 2020.

9. Heo, K. Yun and J. Y. Choi, "Appearance and motion based deep learning architecture for moving object detection in moving camera," 2017 IEEE International Conference on Image Processing (ICIP), 2017, pp. 1827-1831, doi: 10.1109/ICIP.2017.8296597.

10. Liang and F. R. Yu, "Wireless Network Virtualization: A Survey, Some Research Issues and Challenges," in IEEE Communications Surveys \& Tutorials, vol. 17, no. 1, pp. 358-380, First quarter 2015,doi:10.1109/COMST.2014.2352118. 
11. Kampanakis, H. Perros and T. Beyene, "SDN-based solutions for Moving Target Defense network protection," Proceeding of IEEE International Symposium on a World of Wireless, Mobile and Multimedia Networks 2014, 2014, pp. 1-6, doi: 10.1109/WoWMoM.2014.6918979.

12. Basu, G. Moretti, G. Sen Gupta and S. Marsland, "Wireless sensor network based smart home: Sensor selection, deployment and monitoring," 2013 IEEE Sensors Applications Symposium Proceedings, 2013, pp. 49-54, doi: 10.1109/SAS.2013.6493555.

13. Corujo, C. Guimaraes, B. Santos and R. L. Aguiar, "Using an open-source IEEE 802.21 implementation for network-based localized mobility management," in IEEE Communications Magazine, vol. 49, no. 9, pp. 114-123, September 2011, doi: 10.1109/MCOM.2011.6011742.

14. R ,Moorthi.M “Efficient Routing protocols for Mobile Ad Hoc Networks", International conference on AEEICB (978-1-5090-5434-3) IEEE SJR 1.2 August 2017.

15. Kachirski and R. Guha, "Effective intrusion detection using multiple sensors in wireless ad hoc networks," 36th Annual Hawaii International Conference on System Sciences, 2003. Proceedings of the, 2003, pp. 8 pp.-, doi: 10.1109/HICSS.2003.1173873.

16. Kachirski and R. Guha, "Intrusion detection using mobile agents in wireless ad hoc networks," Proceedings. IEEE Workshop on Knowledge Media Networking, 2002, pp. 153-158, doi: 10.1109/KMN.2002.1115178.

\section{Figures}




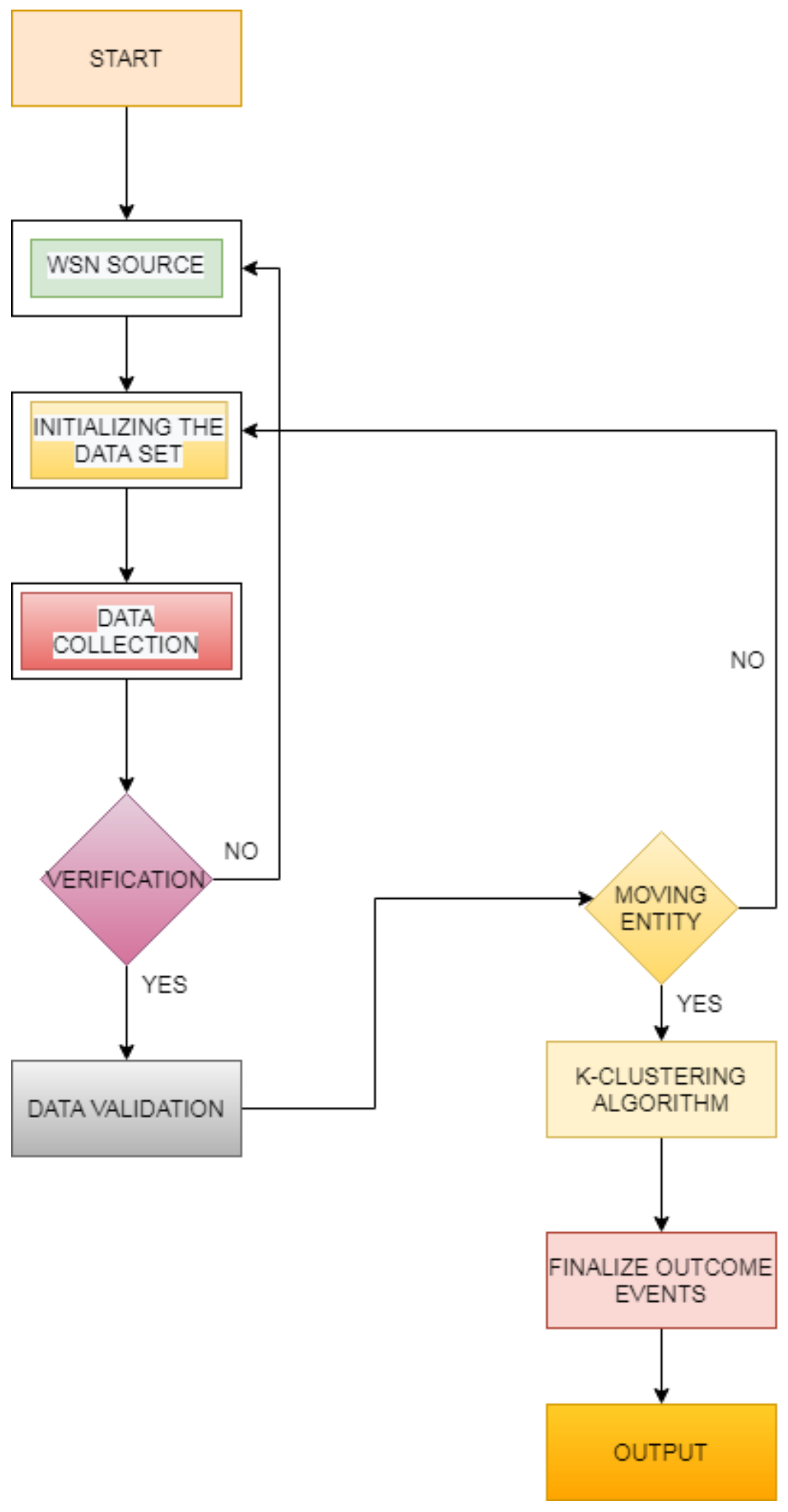

Figure 1

Flowchart Diagram 


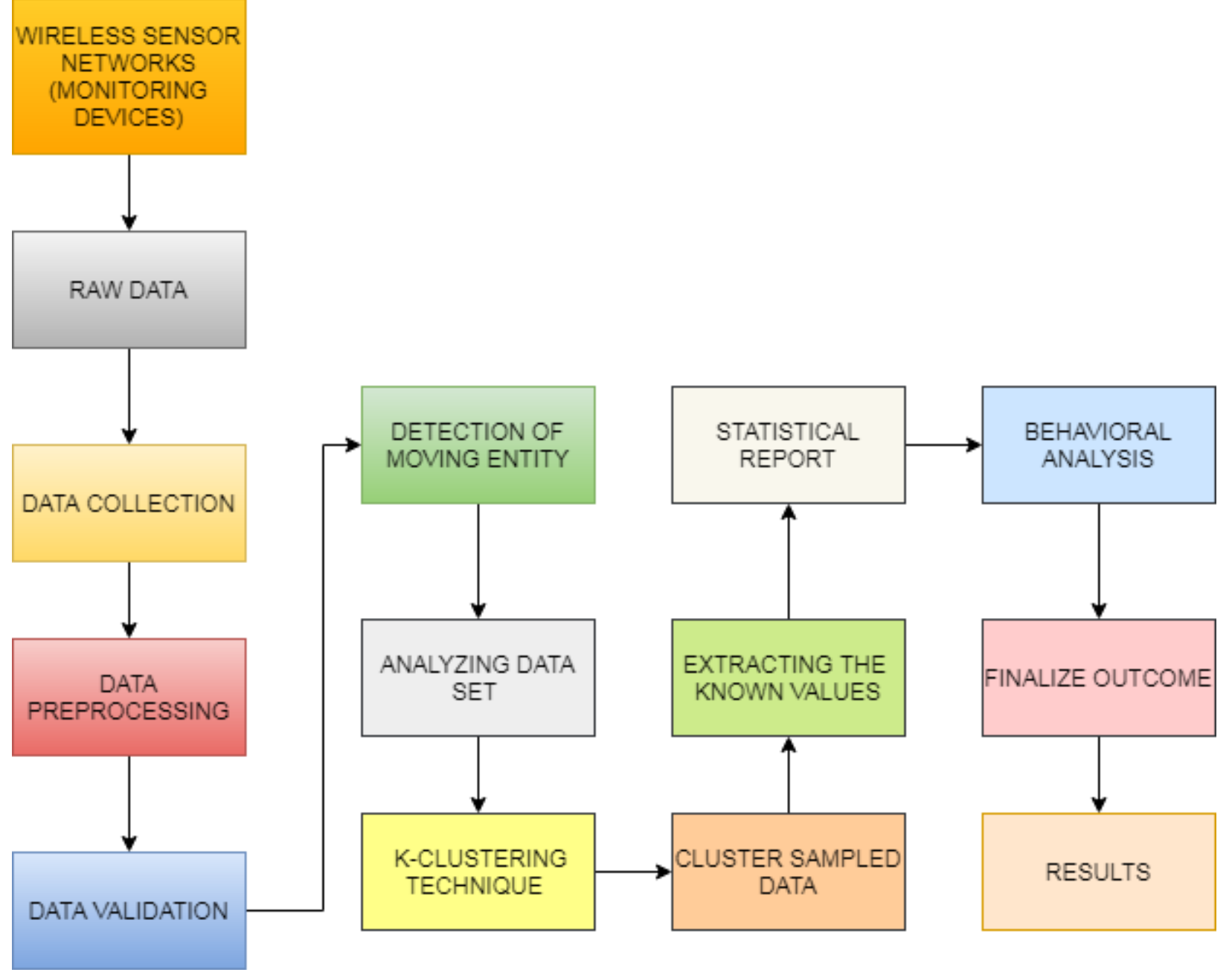

Figure 2

Architecture Diagram

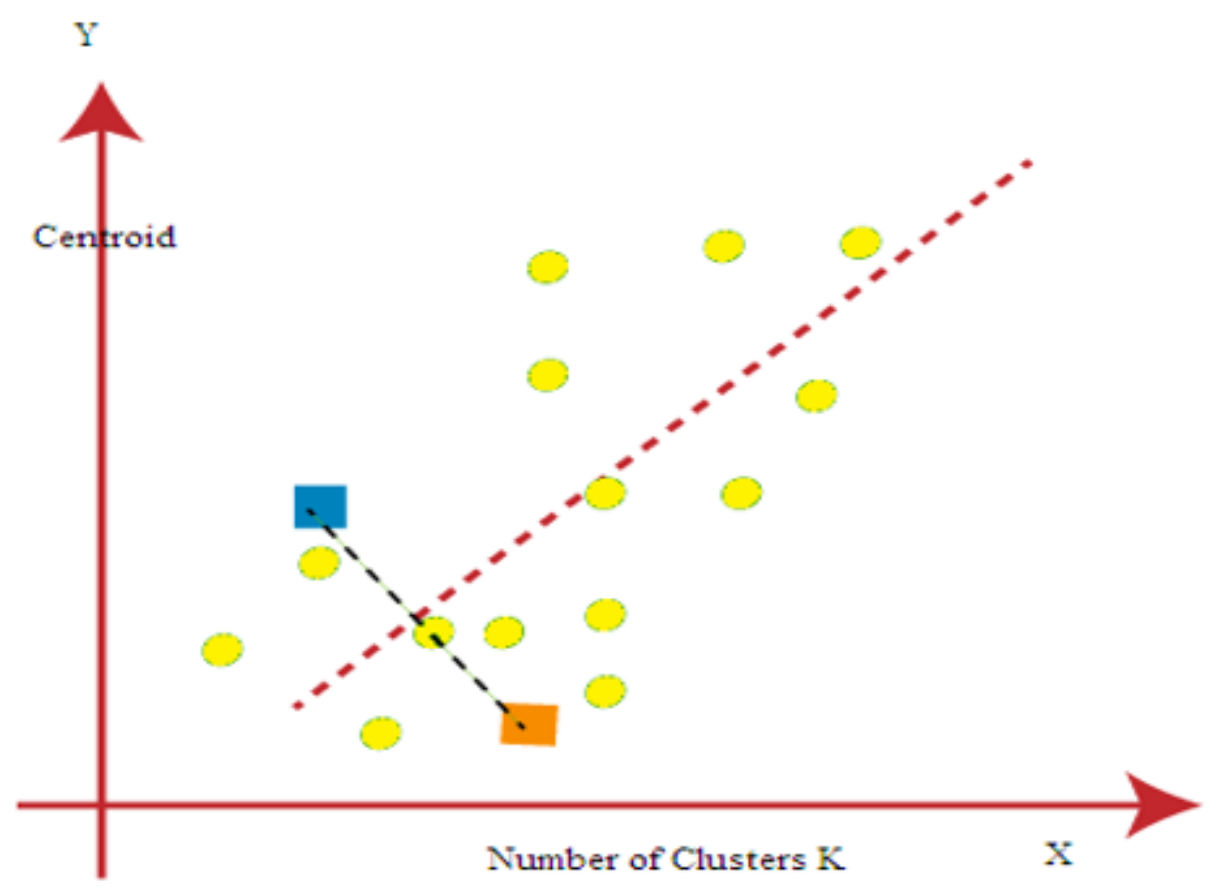


Figure 3

Caption not included with this version.

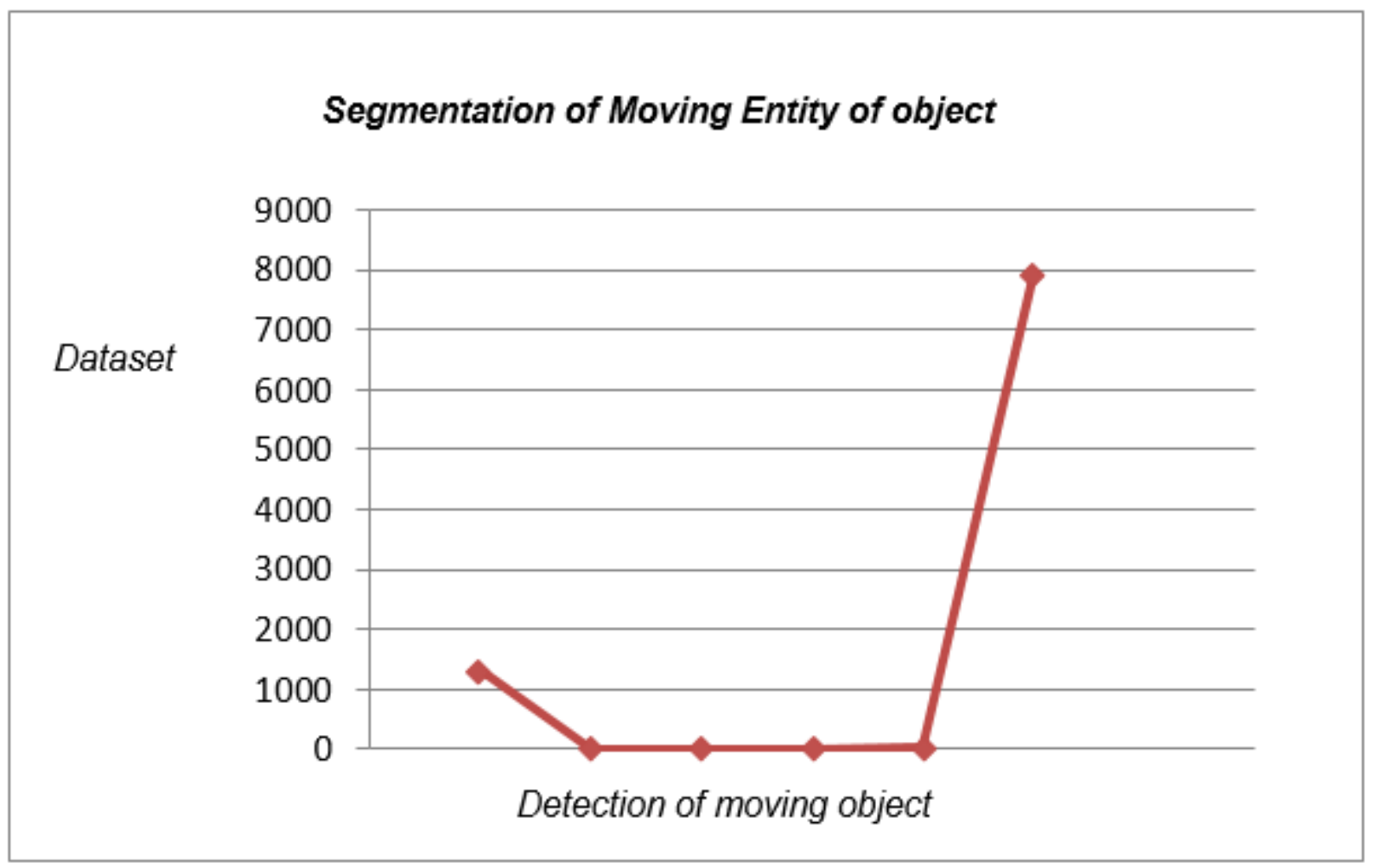

Figure 4

Caption not included with this version. 